Proceedings

\title{
Experiential Simulation and Environmental Psychology for Anticipating Users' Reactions to Design Projects: An Application in Architectural Higher Education ${ }^{+}$
}

\author{
Barbara E. A. Piga ${ }^{1, *}$, Marco Boffi ${ }^{2}$ and Nicola Rainisio ${ }^{2}$ \\ 1 Laboratorio di Simulazione Urbana fausto Curti, Dipartimento di Architettura e Studi Urbani, Politecnico \\ di Milano, 20133 Milano, Italy \\ 2 Dipartimento di Beni Culturali e Ambientali, Università degli Studi di Milano, 20122 Milan, Italy; \\ marco.boffi@unimi.it (M.B.); nicola.rainisio@unimi.it (N.R.) \\ * Correspondence: barbara.piga@polimi.it; Tel.: +39-02-2399-5561 \\ + Presented at the International and Interdisciplinary Conference IMMAGINI? Image and Imagination \\ between Representation, Communication, Education and Psychology, Brixen, Italy, 27-28 November 2017. \\ Published: 20 November 2017
}

\begin{abstract}
The contribution presents an interdisciplinary approach to education developed by the authors, who belong to the field of study of urban design and environmental psychology. The presented study is part of a wider research aiming at developing a methodology for assessing, before construction, the impact on people's well-being generated by urban design projects. The method is based on the combined use of immersive visualization and psychological survey, administered to inhabitants along the design process, for testing - and potentially re-tuning - the architectural solutions. In particular, the paper presents a case study application with a university class of architects and planners of the last year of the Master of Science. The paper outlines the general contents of the ongoing experimental research, it provides a brief theoretical framework, it describes the tools used and developed ad hoc, and it finally illustrates the case study application.
\end{abstract}

Keywords: experiential simulation; environmental psychology; urban design

\section{Introduction}

The contribution presents the development of an ongoing interdisciplinary research conducted by two research units, namely Laboratorio di Simulazione Urbana 'Fausto Curti' (Fausto Curti' Urban Simulation Laboratory) (labsimurb) - Department of Architecture and Urban Studies (DAStU) Politecnico di Milano (POLIMI) and Department of Cultural Heritage and Environment-Università degli Studi di Milano (UNIMI), belonging to the field of simulation for urban design and environmental psychology. The aim of the research is to develop an interdisciplinary methodology for assessing urban design projects before construction. In a nutshell, we propose a combined use of immersive visualization and psychological survey administered to inhabitants of urban transformation areas along the design process. The main goal is to anticipate users' responses to the designed environment for (i) pre-evaluating the well-being generated by urban design solutions; (ii) testing the coherence between design concept and results; and thus informing the design process (iii) supporting negotiation and decision making of public administrations in the urban design and planning field. In particular, this contribution focuses on the application of the methodology to the architectural higher educational domain, and it presents the results of the first experimental application into a class of the last year of the Schools of Architecture and Urban Planning. More in detail, the procedure was applied along the Master of Science course of Architectural and Urban 
Simulation (academic year 2016-2017), prof. B. Piga and prof. R. Salerno, at POLIMI in collaboration with prof. M. Boffi and prof. N. Rainisio from UNIMI.

\section{Ecological Validity: A Brief Research Framework}

In order to produce an effective procedure for assessing people's reactions to a future environment, that of course implies some ethical issues see for instance [1], it is important to rely on solid research outcomes both on the side of simulation and on the one of psychology. We have taken as a main relevant reference the field of experiential simulation see for instance $[2,3]$ and the related ecological validity or response equivalence studies conducted starting from the late sixties by the Environmental Simulation Laboratory at the University of California at Berkeley [2,4-6]. These studies aimed at verifying the correspondence between people's reaction to a real environment and its simulation, through an investigation and assessment on: (i) the simulation as a media (environment fidelity); (ii) the users' reactions to the visualization (interface fidelity) [7]. We started to develop further this studies by investigating people's feedback using new devices, such as Head Mounted Displays $(H M D)$, and by combining a psychological investigation on the well-being generated by the designed urban scenarios.

Focusing on the media per se, it is important rely on the research outcomes that defined the distinctive features of a trustworthy experiential simulation. In fact, in order to produce a reliable media, that is the basis for a credible simulated experience, it is important to take into account several characteristics that guarantee the perceptual fidelity of the user/simulation interaction. Among the others, and according to different authors, simulations should in fact be: realistic, accurate, understandable and unbiased $[1,2,5,6]$. This imply the fact, for instance, that all the elements present in the visual field should be depicted, but also that they should be realistically represented in terms of location in space, scale, layout, details and textures; this should be done taking into account the observer perspective and motion, i.e., the relative (perceived) dimensions, proportions and positions of the elements in space that change in time. 3D modelling and rendering software facilitate the the generation of such simulations, but results are not always reliable; several kinds of distortions can in fact occur, for instance the use of a wide angle lens unrealistically emphasizes falling lines and alters the perceived sense of dimensions and distances in subjective views $[1,8]$. The fidelity of the final results in terms of user engagement and comprehension of the future experience is also influenced by the device used and on the presentation setting. In any case, novel immersive devices, such as HMD, and user friendly software are widening the possibility of applications and the efficacy of experiential simulation, at least in terms of people engagement.

The general framework guiding the psychological contribution to the present approach to architectural higher education is mainly based on the concept of experience. This is a concept deeply rooted in the psychological studies since their origins, fundamental to express the impossibility of separating the environment from the person acting in it [9]; focusing on subjective experience is hence considered the only way to properly explore the interaction between human and environmental factors [10-12]. This implies the refusal of the mechanistic reductionism, in favor of a more holistic description of the reciprocal influence exerted by space, cognition, and social dynamics [13]. Consistently with such phenomenological perspective, we identified some specific psychological constructs which allow to fruitfully integrate the traditional architectural perspective: the Presence Theory [14], the Flow Theory [15] and the Preference Matrix [16]. The combination of approaches is the basis of the research under development by the authors and the framework for the first application of the method for higher education.

The notion of presence can be conceived as an "illusion of nonmediation" [14], occurring when a person is not capable of perceiving or accurately defining the role of a medium in the interaction with the surrounding environment. In such conditions the individual acts as if the medium were not present, living an experience that can be assumed as closest as possible to nonmediated ones, like in everyday life. The focus on the topic increased in the last years, due to the spread of new technologies which significantly favor the possibility of creating smooth and immersive interactions with virtual environments. In the authors' perspective, presence can occur only when a person is using a medium, 
then is the result of the interaction between an individual and a tool. This notwithstanding, since it is conceived as a perceptual illusion, it must be considered as a state exclusively attributable to a person. For our purposes a relevant feature of presence is its complexity, as noted by ljsselsteijn and Riva [17] (p. 3). Hence, it is not simply a reaction, but a complex experience resulting from several cognitive processes and including multi-sensory data.

Csikszentmihalyi [15] described flow as another well-established construct, which can be fruitfully used to define subjective experience. Since its origins it is described as a state in which an individual feels completely involved in an activity, associated to engagement and enjoyment. Initially observed mainly in artists and athletes, it is nowadays recognized as a universal experience resulting from various activities in many different cultural contexts [18]. It is then considered as a complex state, resulting from the interaction of cognitive, motivational and emotional factors; the reciprocal influence of these factors gives origin to a coherent condition, which is considered an optimal experience for individuals because promoting personal development. In such perspective it is often regarded as one of the components conducive to well-being. It cannot be controlled in a voluntary way, but only results when individuals face environmental challenges which are perceived as demanding, even if not too difficult to cope with them. Over the years many efforts have been made to identify different components which can describe more in detail this kind of experience [19]: challenge-skill balance (perceived competence for the task); action-awareness merging (actions are done spontaneously without effort also when including complex tasks); clear goals (to identify specific route of action); unambiguous feedback (regular monitoring of the execution); concentration on the task at hand (ignoring anything not related with the performance); sense of control (feeling of mastery); loss of self-consciousness (oneness with the environment that leaves no space for others' evaluation); transformation of time (altered speed of time); autotelic experience (the positive feeling experienced is the main reward for the activity).

In the field of environmental preference, Kaplan and Kaplan's theory [16] has been taken as a reference, since on the one hand it is one of the most widely accepted in literature, and on the other hand it is a virtuous mediation between evolutionary theory and a cognitive approach. Moreover, it is fundamentally related to the theoretical patrimony of design science, as it shares with it the relevance attributed to certain environmental features (i.e., complexity, legibility), which are also present in the designers' way of thinking when looking at a place and its formal qualities. The theory is based on the recognition of two primary cognitive needs in humans: comprehension, namely the ability to interpret the context to facilitate adaptation, and exploration, which is the need to constantly expand our knowledge, always for survival purposes. Is the perceived balance between these two poles, according to Kaplan and Kaplan, to generate a higher environmental preference rating. In fact, if a place is too unbalanced in favor of comprehensibility, it will be lacking in that "promise of new information" which stimulates interest and curiosity. Conversely, if it is too unbalanced on the exploration pole, it will be poorly suited to our cognitive schemata and then anxiogenic. The two authors interlace this vision with a theory of knowledge based on two steps: immediate and inferred. The first step corresponds to the gestaltic impressions generated by the first visual contact with an environment, whereas the second refers to the inferences that human beings make from that first impression in terms of possibilities of action. The final result is a preference matrix (knowledge steps $X$ cognitive needs) consisting of four factors: coherence and legibility (comprehension) and complexity and mystery (exploration). These factors are the basic elements of environmental preference, which is determined by the dynamic relationship between them.

\section{The Main Tools}

From theory to practice there is always a gap due to different reasons. One of the major ones is due to resources at hand and time constraints. Our experimental study was not an exception. For this reason, we decided to combine very simple tools for producing simulations. In fact we designed the 3D urban model in SketchUp ${ }^{\circledast}$ by Trimble ${ }^{\circledR}$ (Sunnyvale, CA, USA), where we applied post-processed photographs of the existing buildings' facades, and later we imported it into the rendering software Lumion ${ }^{\circledR}$ by Act-3D ${ }^{\circledR}$ (Warmond, The Netherlands, EU) for the texturing and rendering of the final 
visualizations (Figure 1). We decided to use this tools because they are easy to learn and to use despite enabling high quality results in a short time. The limit of this choice is mainly related to the visualization style of Lumion ${ }^{\circledast}$, that is peculiar and recognizable and produces visualizations that are too clean and pleasant. The modification of this fancy style is difficult to avoid, and this of course can incur a sort of bias if abused. For this reasons we tried to control this effect and we embedded the styles in the model as a reference for students. With this software it is very easy and fast to save spherical panoramas that can be presented using an HMD. Even in the choice of these devices we followed the same strategy as for software; we used cheap Cardboard where it is possible to insert smartphones of different sizes for visualizing the simulations in an interactive way. The same panorama produced for HMD can be visualized and navigate directly in the browser of the desktop pc or of mobiles. It is important to remark that the use of this tools has been intended as iterative to support the typical non-linear design process for more details on the approach adopted see [3].

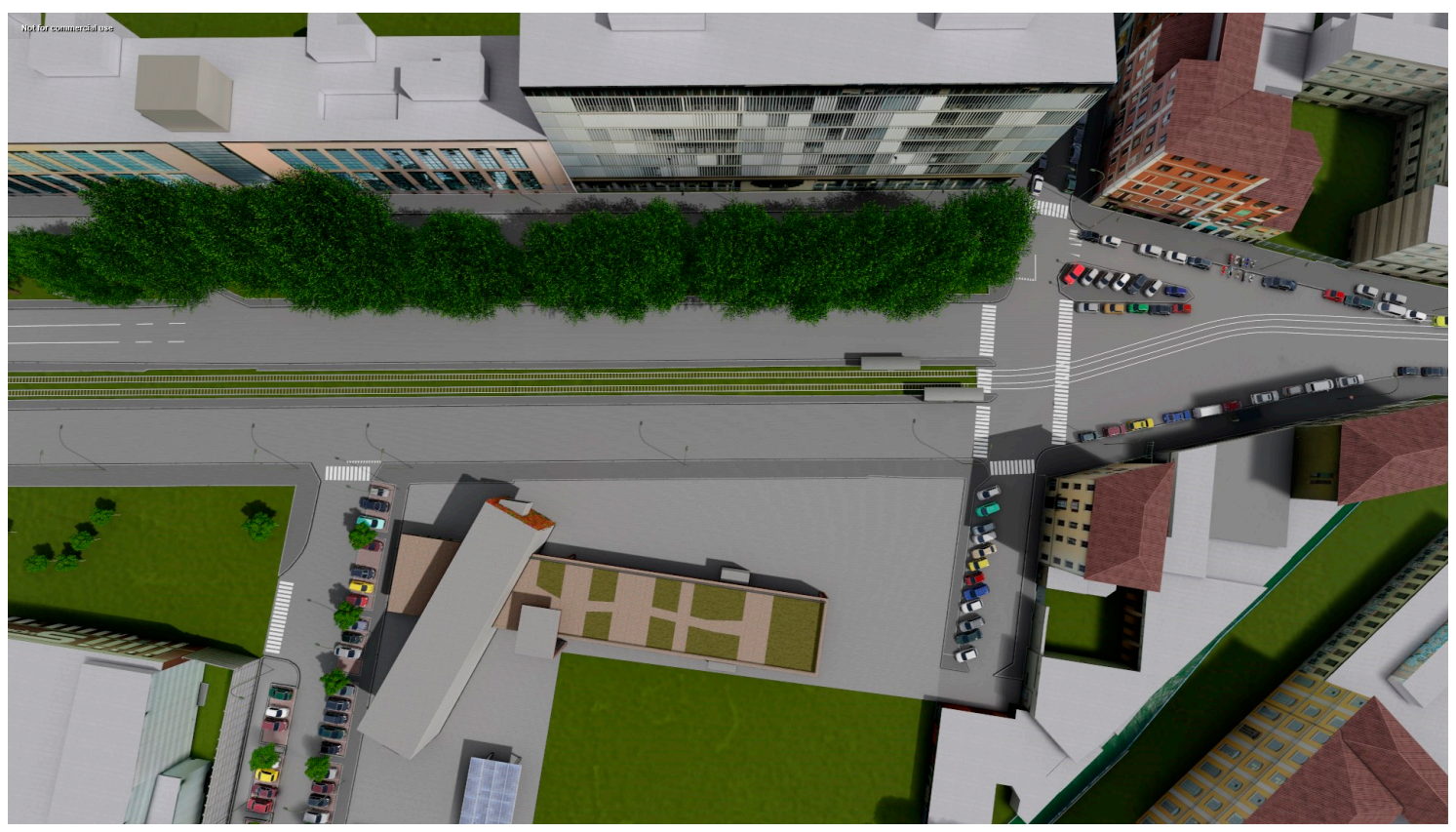

Figure 1. Render of the 3D model provided to students.

The other tool used is the environmental survey we designed, namely EPSUD (Environmental Psychological Survey for Urban Design), that is an outcome of a wider research and that was prepared ad hoc for this type of investigation. It is structured for including architectural and psychological aspects considered relevant for describing the human-environmental interaction.

The survey included different parts:

- $\quad$ an introduction aimed at identifying the single architectural project explored and the medium to do it (e.g., computer screen or cardboard);

- the description of the environment through adjectives and activities;

- items designed to explore the concept of environmental preference and items regarding flow experienced in that specific environment, built relying on the literature described above $[15,16]$ and the Flow in Environment Scale [13]

- the Independent Television Company Sense of Presence Inventory (ITS-SOPI) developed by Lessiter et al. [20]. The questionnaire is divided into two parts, which include items describing the impressions experienced during and immediately after the interaction with the medium. The underlying factors are: Sense of Physical Space, Engagement, Ecological Validity, and Negative Effects;

- socio-demographic data about the respondent, including some information regarding the knowledge of the neighborhood and the area of residence. 
According to the procedure students were invited to show their project to voluntary people and then administer the whole questionnaire using an online platform.

\section{The Milan Smart City Lab: A Case Study Application}

In the course of Architectural and Urban Simulation we tested the use of such an approach with the class. Students from the courses of study of architecture and planning developed a design project for the open area behind the Smart City Lab of the Municipality of Milan, in via Ripamonti 88, that will be built in 2018 (Figure 2). This will be a new public facility located in the south Milan, that will mainly host temporary co-working activity and will be an incubator for startups dealing with smart and sharing city solutions. The Smart City Lab and its open space will cover a total area of around 2 Ha; since this is a public facility, students were allowed to conceive the entire land as a public service.

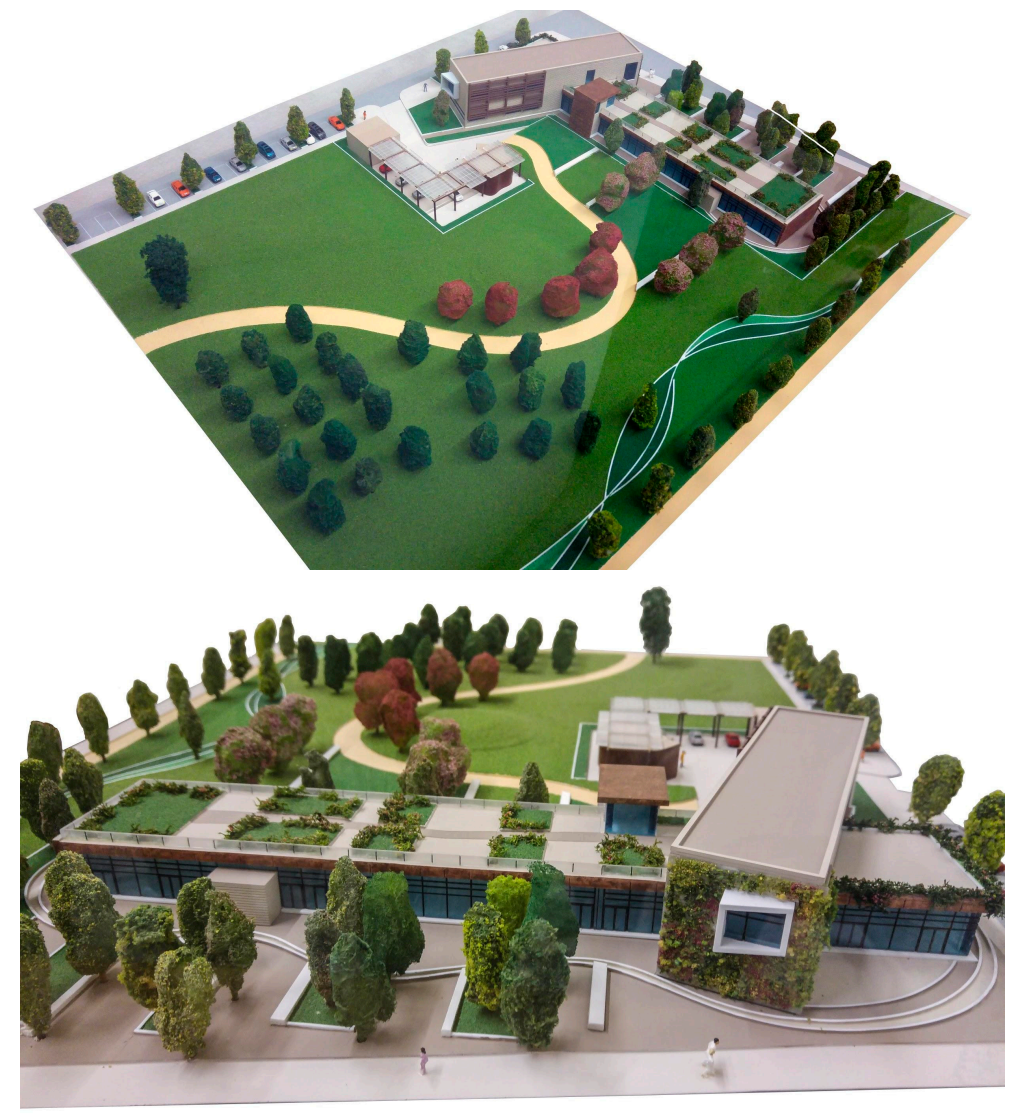

Figure 2. Physical model of the design project of the Milan Smart City Lab. The building is now in the executive design phase, while the open space still do not have a defined design project.

The class was stimulated to develop design solutions strongly focused on multi-sensory experiences addressed to the specifics targets of population they had to individuate. Beyond the direct observation of the area and its livability, the entire urban design project was developed referring to the provided photorealistic 3D digital models of the context made with SketchUp ${ }^{\circledast}$ and Lumion ${ }^{\circledast}$. Along the entire process we asked students to navigate the 3D model using a subjective perspective in order to discover and investigate relevant views and paths of the area, as it is today, or to test the design alternative solutions from a pedestrian perspective. We believe that this is a crucial process for supporting design conception based on the user experience of the place in motion. We set up the model providing some pre-defined relevant points of view in order to stimulate the entire class to consider that perspectives as important ones. Each group was supposed to produce spherical panoramas from these pre-fixed locations besides others relevant views they had to identify according to their specific design concepts and schemes. Students visualized the site using monitors (non-immersive visualization) and Head Mounted Displays (immersive visualization), specifically 
Cardboards that were provided to each group. In so doing we invited groups to tackle the design process with an experiential-even if mainly visual-approach, e.g., identifying existing or proposed landmarks, relevant routes and relative activities, and so on. In parallel with the theoretical lessons on urban design and simulation, students were trained to use the rendering software, and use it as a design tool and not only as a final presentation device, as they were often used to do. The process of exploring subjective views, of the existing condition or design solution, was intended as a recursive process of trial and error [3]. Of course, the 3D model contributes to focus students' attention on the visual and kinesthetic senses alone. No doubts that this imply a relevant lack of the other sensory spheres that might lead non-expert professionals, such as students, to avoid thinking in a multisensory way. Unfortunately experiential simulation that includes a reliable anticipation of sounds and other sensory dimensions of a future environment is still hard to produce in an easy and accurate way. So we simply asked students to take into considerations other senses and to represent them using different non-experiential representation modalities that accompanied the immersive simulations. Anyhow, in order to stem the mere visual centric approach, we stimulate students to conceive and design the proposal starting from the evolving in motion and multisensory experience.

In addition to the main part of the course, mainly dedicated to experiential simulation for environmental design, the psychologist M. Boffi and N. Rainisio traced a parallel path focusing on the relationship between psychological experience and urban design. First of all, some theoretical fundamentals in environmental and social psychology were briefly introduced, enabling our students to understand the importance of a people-centered approach to design. The main theories on environmental preference were presented, together with the constructs of sense of presence and flow, in order to properly explore the complexity of the environmental experience; it also allowed to adequately present the survey to the students and discuss the results for each project assessed. Secondly, general methodologies and research tools were introduced, focusing in particular on the connection between the survey and the outlined theoretical aspects. Students were also trained on the main strategies to design and administer a field questionnaire, and each group had to collect 30 completed questionnaires, to reach an overall total of 180. Moreover, before administering the survey, we asked students to fill in the same survey according to their design desiderata or, in other terms, according to their expectations and envisioning of inhabitants' reactions to the designed environment. This process had a twofold goal. On one side it stimulated groups to clearly state their design desiderata; on the other side, it was the reference of comparison to understand the possible gap between desiderata and inhabitant's assumable reactions. Each group had to collect surveys after a visualization task performed with HMDs. We also recommended to select their respondents between people not involved in the project and living in the area, to discourage possible bias due to a familiar proximity to the interviewer or to a lack of knowledge about the urban context respectively.

In the third and final phase, the professionals M. Boffi and N. Rainisio (Figure 3) analyzed the collected results and presented these to students. For each project, the compatibility/incompatibility with the desiderata, and which individual or collective behaviors the designed affordances tended to elicit/discourage have been highlighted. Furthermore, the perceived elements of strength and weakness in terms of place attachment, probability of generating optimal experiences, and matching between individual cognition and place (legibility, complexity) have been evaluated.

By highlighting the gap between expectations and reactions of the inhabitants, students had a third point of view, beyond the professors' one, that contributed to inform them on the efficacy of the design solution according to their desiderata. The collegial discussion of results, and the clarification of the specific link between people's reaction and the urban design solution they adopted, powered a fruitful discussion between professors and the class in an interdisciplinary perspective. Moreover, this process supported the rebalancing of the attention of students on the human/environment interaction rather than the architectural artifact per se. Finally yet importantly, the process increased the confidence of students towards teachers, since the comments received during the reviews coincided with the feedback they had with the users, and of course professors were able to support them in understanding the weakness and potentiality of the draft proposal towards a more effective design solution. 


\section{MAIN VARIABLE OVERVIEW}

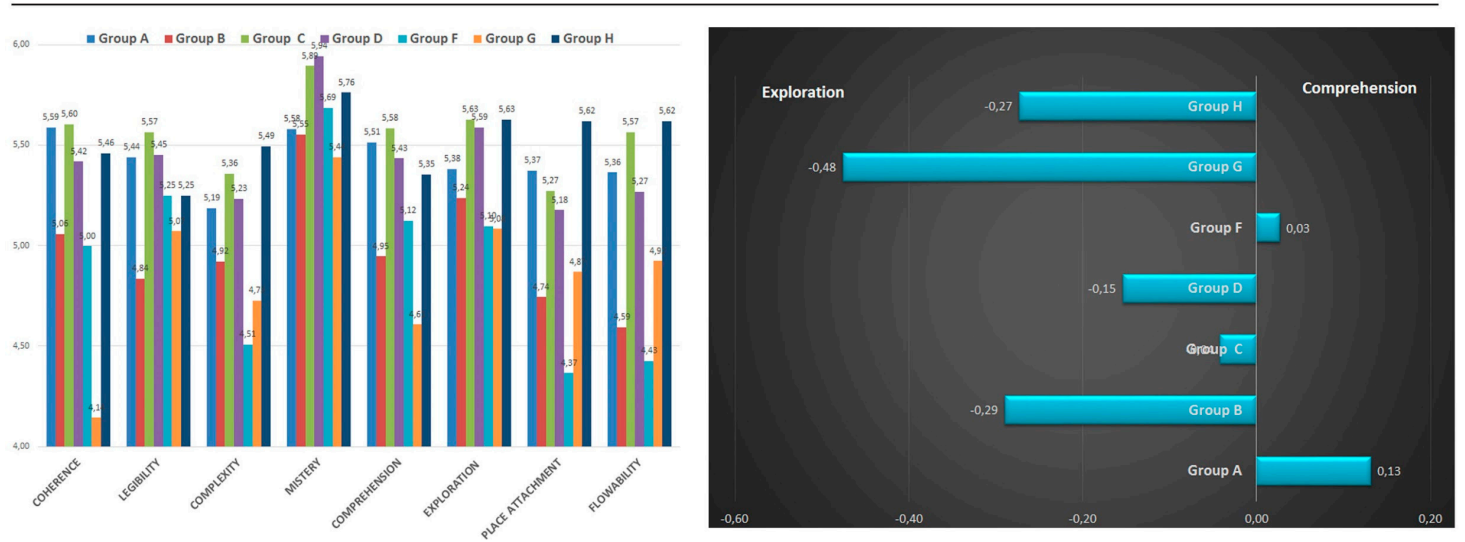

Figure 3. Two histograms of the analysis developed by the psychologists M. Boffi and N. Rainisio based on the collected surveys by groups.

\section{Conclusions}

Even if we are aware that it impossible to produce a perfect crystal ball, we believe that the possible failure-and relative impact on cities and inhabitants-of design projects, and hence the evaluation of risks, can be fruitfully supported by this approach. Moreover, the interdisciplinary process enable students to deeper understand the contributions that other disciplines can give to the design one and vice versa. Moreover, the use of render software and device that were not known by groups were an engaging trick to involve them more deeply into the subject. Their interest was also supported by the fact that the same representation tools could have been used in other courses or later in the professional practice. Last but not least, the easiness of usage of software and devices favoured a recursive experiential design workflow.

The spontaneous comparison between teachers' remarks on the design project and the interpretation of the reactions by future inhabitants increased the confidence in teachers' reviews, and even stimulate questions and fruitful discussions on the reasons why the group expectations were or were not accomplished by inhabitants, i.e., the gap of what students had in mind and what they designed.

At the end of the course the comments by students on the entire interdisciplinary experience were absolutely favorable and during the final presentation of the design solution more than once they rely on the inhabitants' feedback for defending the final design solution adopted (Figure 4). We hope that this approach would reinforce student's design attitude based on people living the environment rather than the architectural object as an artifact per se. 


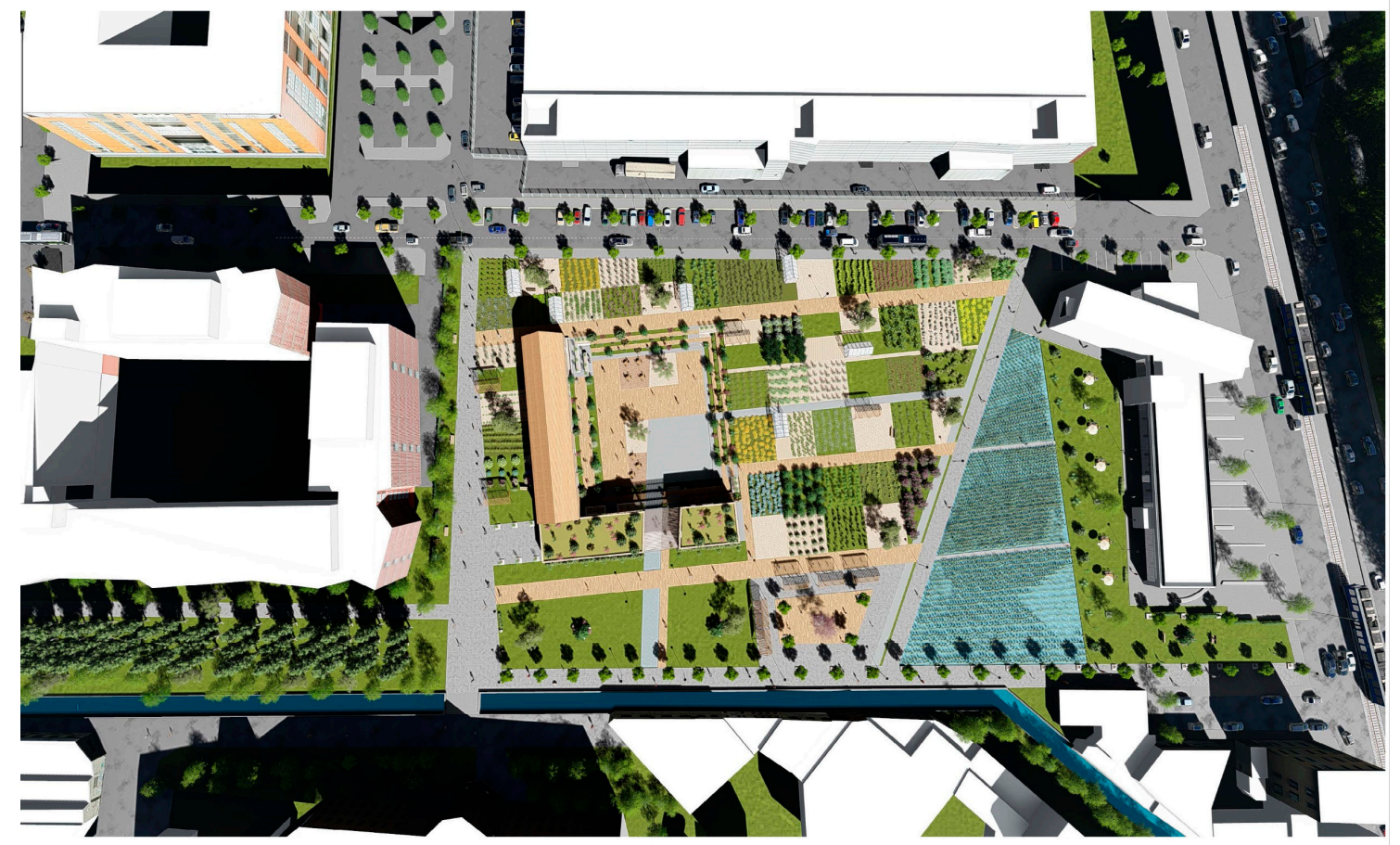

Figure 4. General overview of the design solution, named "Smart Farm", developed by the group composed by: Erze Dinamara, Jake Mace, Luis Felipe Mata Munguia, Borbala Papp, Angela Daniela Alvarez Sussoni, Chandi Vaghasia, Austra Kakeseva, Weronika Wojtkowiak (video at: http://bit.ly/2vT719D).

Author Contributions: Part 1 was developed by B. Piga; the other parts were developed by B. Piga concerning the experiential simulation topic, and by M. Boffi and N. Rainisio regarding the psychological assessment. The combined methodology was developed by the three authors together with an interdisciplinary approach.

Conflicts of Interest: The authors declare no conflicts of interest.

\section{References}

1. Sheppard, S.R.J. Visual Simulation: A User Guide for Architects, Engineers, and Planners; Van Nostrand Reinhold: New York, NY, USA, 1989.

2. Piga, B.; Morello, E. Environmental Design Studies on Perception and Simulation: An Urban Design Approach. Ambiances Environnement Sensible Architecture et Espace Urbain 2015, doi:10.4000/ambiances.647, Available online: http://ambiances.revues.org/647 (accessed on 17 November 2017).

3. Piga, B. Experiential Simulation for Urban Design: From Design Thinking to Final Presentation. In Urban Design and Representation, A Multidisciplinary and Multisensory Approach; Piga, B.E.A., Salerno, R., Eds.; Springer International Publishing: Cham, Switzerland, 2017; pp. 23-36.

4. Appleyard, D.; Craik, K.H.; Klapp, M.; Kreimer, A. The Berkeley Environmental Simulation Laboratory: Its Use in Environmental Impact Assessment; IURD - Institute of Urban and Regional Development (Working Paper); University of California: Berkeley, CA, USA, 1973; Volume 203, p. 21.

5. Appleyard, D. Understanding Professional Media. In Human Behavior and Environment; Altman, I., Wohlwill, J.F., Eds.; Plenum Press: New York, NY, USA, 1977; pp. 43-88.

6. Bosselmann, P. Representation of Places: Reality and Realism in City Design; University of California Press: Berkeley, CA, USA; Los Angeles, CA, USA; London, UK, 1998.

7. Waller, D.; Hunt, E.; Knapp, D. The Transfer of Spatial Knowledge in Virtual Environment Training. Presence 1998, 7, 129-143.

8. Piga, B. La Simulazione Visiva per L'urbanistica. Il Punto di Vista Percettivo Nella Comprensione Delle Trasformazioni Urbane. Ph.D. Thesis, Politecnico di Milano, Milan, Italy, 2010.

9. Ittelson, W.H. Environment and Cognition; Seminar Press: New York, NY, USA, 1973.

10. Ryan, R.M.; Deci, E.L. On happiness and human potentials: A review of research on hedonic and eudaimonic well-being. Annu. Rev. Psychol. 2001, 52, 141-166. 
11. Joye, Y.; van den Berg, A. Is love for green in our genes? A critical analysis of evolutionary assumptions in restorative environments research. Urban For. Urban Green. 2011, 10, 261-268.

12. Boffi, M.; Rainisio, N. To Be There, Or Not to Be. Designing Subjective Urban Experiences. In Urban Design and Representation; Piga, B.E.A., Salerno, R., Eds.; Springer International Publishing: Cham, Switzerland, 2017; pp. 37-53.

13. Rainisio, N.; Boffi, M.; Riva, E. Positive change in environment: Aesthetics, environmental flowability and well-being. In Enabling Positive Change: Flow and Complexity in Daily Experience; Inghilleri, P., Riva, G., Riva, E., Eds.; De Gruyter: Warsaw, Poland; Berlin, Germany, 2014; pp. 91-104.

14. Lombard, M.; Ditton, T. At the heart of it all: The concept of presence. J. Comput.-Mediat. Commun. 1997, 3, doi:10.1111/j.1083-6101.1997.tb00072.x.

15. Csikszentmihalyi, M. Beyond Boredom and Anxiety; Jossey-Bass: San Francisco, CA, USA, 1975.

16. Kaplan, R.; Kaplan, S. The Experience of Nature: A Psychological Perspective; Cambridge University Press: New York, NY, USA, 1989.

17. Ijsselsteijn, W.A.; Riva, G. Being There: The experience of presence in mediated environments. In Being There: Concepts, Effects and Measurements of User Presence in Synthetic Environments; Riva, G., Davide, F., Ijsselsteijn, W.A., Eds.; Ios Press: Amsterdam, The Netherlands, 2003; pp. 3-16.

18. Delle Fave, A.; Massimini, F.; Bassi, M. Psychological Selection and Optimal Experience Across Cultures: Social Empowerment through Personal Growth; Springer: New York, NY, USA, 2011.

19. Csikszentmihalyi, M.; Csikszentmihalyi, I. (Eds.) Optimal Experience: Psychological Studies of Flow in Consciousness; Cambridge University Press: New York, NY, USA, 1988.

20. Lessiter, J.; Freeman, J.; Keogh, E.; Davidoff, J. A Cross-Media Presence Questionnaire: The ITC Sense of Presence Inventory. Presence Teleoper. Virtual Environ. 2011, 10, 282-297.

(C) 2017 by the authors. Licensee MDPI, Basel, Switzerland. This article is an open access article distributed under the terms and conditions of the Creative Commons Attribution (CC BY) license (http://creativecommons.org/licenses/by/4.0/). 